\title{
August 2015 Pulmonary Case of the Month: Holy Sheep
}

\author{
Jennifer M. Hall, DO \\ David M. Baratz, MD
}

Banner University Medical Center Phoenix

Phoenix, AZ

\section{History of Present IIIness}

A 42-year-old woman presented to the emergency department with chest pain and dyspnea. The onset of symptoms was acute, initially endorsing left-sided sharp chest pain which then progressed with dyspnea. Chest radiograph was read as normal. Laboratory evaluation was notable for an elevated D-Dimer which prompted a thoracic CT scan to be obtained.

\section{Past Medical History, Family History, Social History}

- She had well-controlled rheumatoid arthritis (on no medical therapy) and was diagnosed with emphysema by her PCP two years earlier.

- Her mother died from pulmonary embolism secondary to underlying lung cancer

- She quit smoking 2 years ago with a total of 20-pack-years.

\section{Physical Examination}

Patient was in mild distress with heart rate of 105, respiratory rate of 22, but otherwise stable, SpO2 was 95\% while breathing ambient air. She had diminished breath sounds in both bases, but otherwise her chest was clear to auscultation. The remainder of the exam was unremarkable.

\section{Radiography}

A chest x-ray (Figure 1) and a thoracic CT scan (Figure 2) were performed.

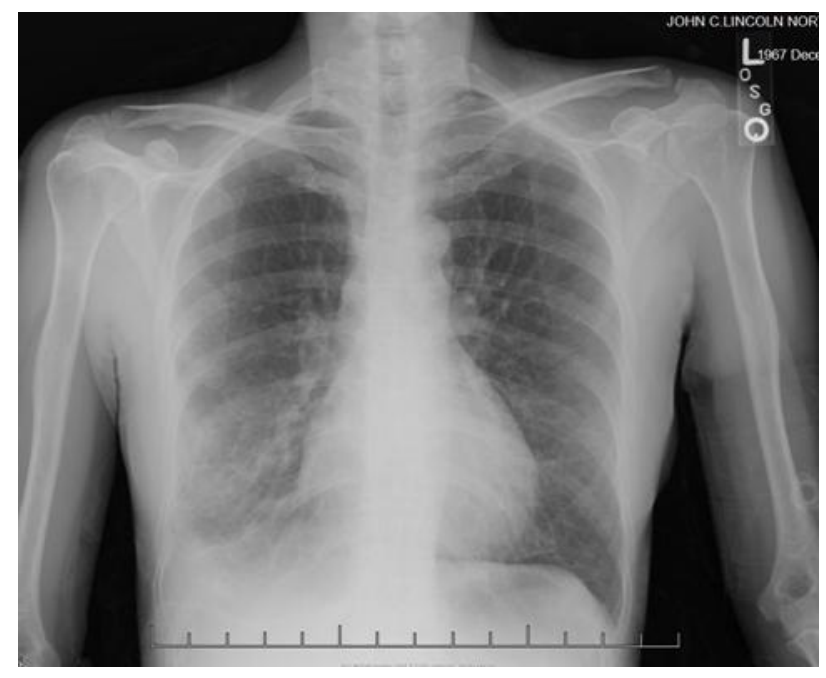

Figure 1. Initial PA of the chest. 


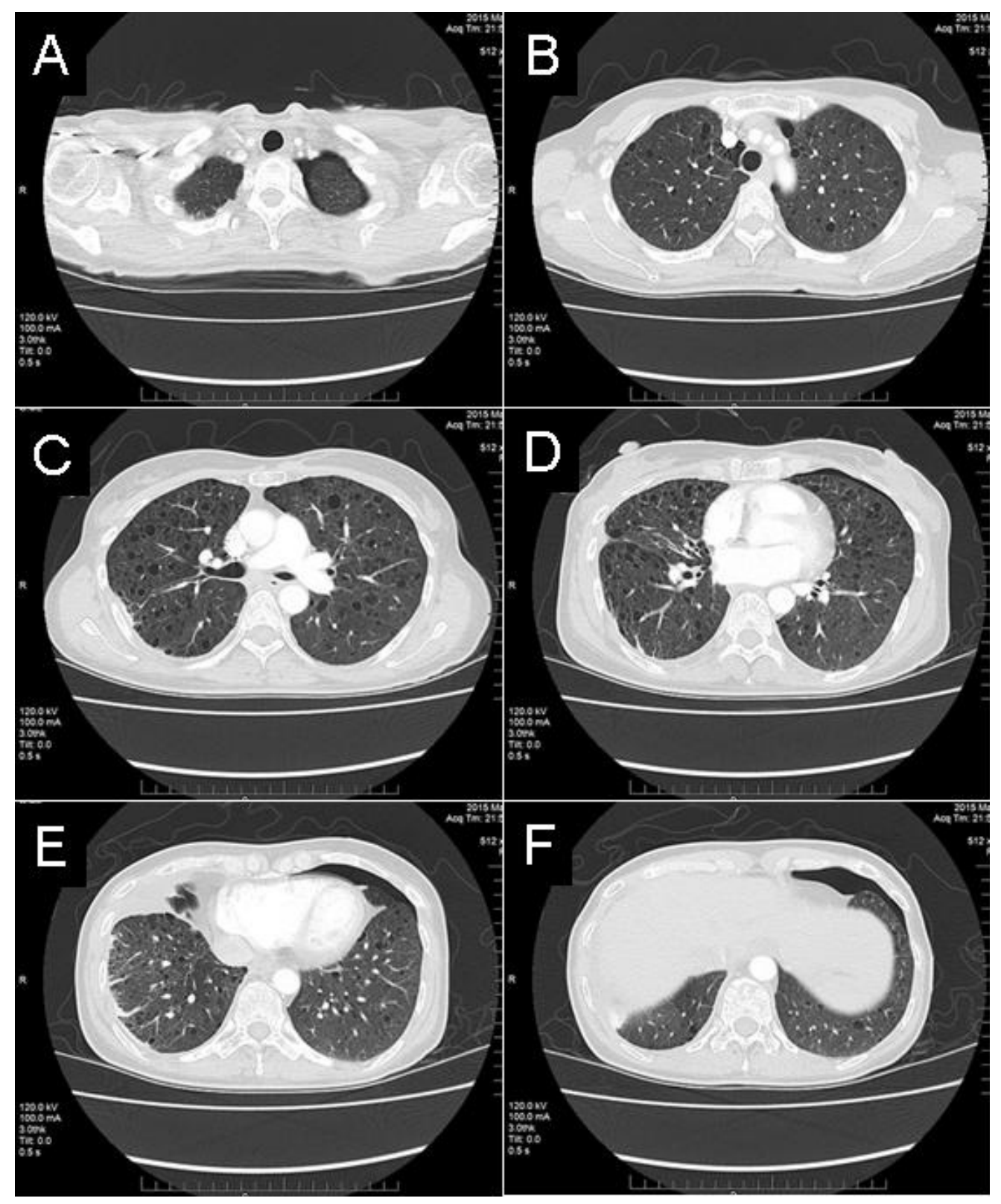

Figure 2. Thoracic CT scan in lung windows.

A chest tube was placed for the left-sided pneumothorax.

What is the next step in management?

1. Bronchoscopy

2. Consider pleurodesis

3. Observation with repeat CT in 3 months

4. Open lung biopsy

5. 1 and 3

6. 2 and 4 


\section{Correct!}

\section{2 and 4}

The thoracic CT shows multiple lung cysts. Rupture of one of these cysts likely led to her pneumothorax. In the setting of cystic lung disease, in order to obtain definitive diagnosis, one should obtain confirmation of the specific type of cystic lung disease, as this will dictate treatment $(1,2)$. Additionally, as she will be at increased risk for recurrent pneumothoraces, consideration should be given to pleurodesis to reduce to chance of recurrence.

The patient underwent VATS with open lung biopsy (Figure 3).

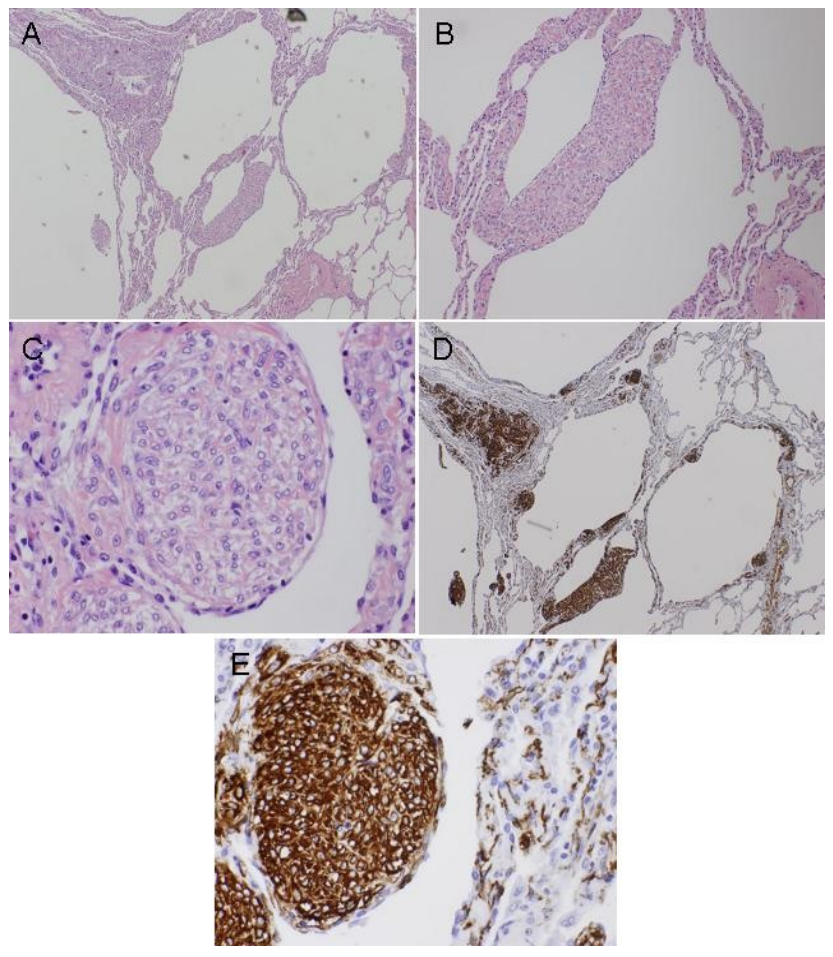

Figure 3. Panel A: $40 \times \mathrm{H}$ \& $E$ showing foci of smooth muscle cell infiltration of lung parenchyma, airways, lymphatics, and blood vessels associated with thin-walled cystic changes. Panel B: $100 \times$ H \& E. Panel C: $400 \times$ H \& E showing small spindle shaped cells and cuboid epithelial cells. Panel D: $40 \times$ smooth muscle actin stain (SMA). The cells stain positive to SMA and human melanoma-black 45 stain. Panel E: 400 x SMA stain.

What is the diagnosis?

1. Birt-Hogg-Dubé syndrome

2. Bronchioalveolar cell carcinoma (mucinous-type invasive adenocarcinoma)

3. Cystic fibrosis

4. Histiocytosis $X$

5. Lymphangioleiomyomatosis 


\section{Correct! \\ 5. Lymphangioleiomyomatosis}

Lymphangioleiomyomatosis (LAM) is an uncommon disease, female-predominant, that is a type of cystic lung disease that has characteristic diffuse thin-walled cysts (1). This disease is caused by the neoplastic growth of smooth muscle cells and spreads via the lymph and hematogenously. Pathology exhibits characteristic infiltration of HMB-45 LAM cells with a smooth muscle phenotype. Inheritance can be autosomal dominant (tuberous sclerosis-LAM) or sporadic (S-LAM), with some males being affected, solely with the TSC-LAM type. Pneumothoraces are common with average age of the first pneumothorax around 35 years of age and a recurrence rate of approximately $75 \%$.

The patient did have small right-sided pleural effusion. Given her known diagnosis now of LAM, what would be the most likely finding on pleural fluid analysis?

1. Cholesterol of $200 \mathrm{mg} / \mathrm{dL}$

2. Glucose of $20 \mathrm{mg} / \mathrm{dL}$

3. $\mathrm{LDH}$ of $30 \mathrm{U} / \mathrm{L}$

4. $\mathrm{pH}$ of 7.10

5. Triglycerides of $150 \mathrm{mg} / \mathrm{dL}$ 


\section{Correct! \\ 5. Triglycerides of $150 \mathrm{mg} / \mathrm{dL}$}

Pleural fluid triglycerides of $>110 \mathrm{mg} / \mathrm{dL}$ is diagnostic of a chylothorax which develops when lipids from chyle collect in the pleural space, usually as a result of injury to the thoracic duct or other regional lymphatic tributaries. Such disruptions can either be traumatic or non-traumatic. As in this case, a LAM-induced chylous effusion is nontraumatic and caused by lymphatic disruption from proliferation of atypical smooth muscle cells and the lymphangetic spread.

After biopsy-proven lymphangioleiomyomatosis was diagnosed and with mechanical pleurodesis performed on the left, what would be the best therapeutic intervention to offer this patient?

1. Cladribine

2. Corticosteroids

3. Endothelin receptor antagonist

4. Rapamycin (sirolimus)

5. There are no established therapies for this disease 


\section{Correct! \\ 4. Rapamycin (sirolimus)}

Treatment for LAM in the past has included hormonal manipulation with the use of antiestrogens, progesterones and /or oophorectomy, but with variable results $(1,2)$. Lung transplantation has also been performed for LAM, although disease recurrence has been documented. Recently, sirolimus, acting via suppression of mammalian target of rapamycin (mTOR) signaling, has been approved by the FDA for the treatment of LAM. mTOR is a protein kinase that regulates cell growth, cell proliferation, cell motility and cell survival. It also causes an increased expression of VEGF (vascular endothelial growth factor), specifically VEGF-C and VEGF-D. The mTOR causes expression of the metastasis, thus promoting lymphangiogenic vascular endothelial growth factors. VEGF-D serum levels are elevated in $50-70 \%$ of patients with LAM. Rapamycin acts to suppress the mTOR, resulting in decreased VEGF levels, hence the mechanism of this treatment. If lung transplant is combined with rapamycin therapy, results may be even more promising. Cladribine is used for pulmonary Langerhans cell histiocytosis (C is incorrect). Corticosteroids can be used to treat lymphoid interstitial pneumonia ( $D$ is incorrect). Endothelin receptor antagonists are used for Group I pulmonary arterial hypertension (PAH). PAH may be seen in patients with LAM, but this is classified as a Group V PAH. This is a result of the LAM cells crossing over from the lymphatics in the thoracic duct into the left subclavian vein and ultimately infiltrating the pulmonary microvasculature, resulting in pulmonary hypertension.

\section{References}

1. Gupta N, Vassallo R, Wikenheiser-Brokamp KA, McCormack FX. Diffuse Cystic Lung Disease, Part I. Am J Respir Crit Care Med. 2015;191(12):1354-1366. [CrossRef] [PubMed]

2. Gupta N, Vassallo R, Wikenheiser-Brokamp KA, McCormack FX. Diffuse Cystic Lung Disease, Part II. Am J Respir Crit Care Med. 2015;192(1):17-29. [CrossRef] [PubMed] 